Mathematical Sciences And Applications E-Notes

Volume 3 No. 1 PP. 108-117 (2015) @ MSAEN

\title{
WELL POSEDNESS OF FIXED POINT PROBLEM FOR MAPPINGS SATISFYING AN IMPLICIT RELATION IN $G_{p}$ - METRIC SPACES
}

\author{
VALERIU POPA AND ALINA-MIHAELA PATRICIU
}

(Communicated by Ishak ALTUN)

\begin{abstract}
The purpose of this paper is to prove a general fixed point theorem in $G_{p}$ - metric space for mappings satisfying an implicit relation. If $G_{p}$ - metric is symmetric, we prove that the fixed point problem is well posed.
\end{abstract}

\section{INTRODUCTION}

In [13], [14], Dhage introduced a new class of generalized metric spaces, named $D$ - metric spaces. Mustafa and Sims [25], [26] proved that most of the claims concerning the fundamental structures on $D$ - metric spaces are incorrect and introduced an appropriate notion of generalized metric space, named $G$ - metric spaces. In fact, Mustafa, Sims and other authors [1], [17], [20], [21], [22], [23], [24], [25], [26], [27], [39], [40], [41], [42], [43], [44] studied many fixed point results for self mappings in $G$ - metric spaces under certain conditions.

In 1994, Mathews [20] introduced the concept of partial metric space as a part of study of denotional semantics of data flows and proved the Banach contraction principle in such spaces. Recently, in [2], [5], [9], [16], [17] and in other papers, some fixed point theorems under various contractive conditions in partial metric spaces are proved.

Quite recently, Zand and Nezhad [46] introduced a generalization and unification of $G$ - metric space and partial metric space, named $G_{p}$ - metric space. In [8], some fixed point theorems in $G_{p}$ - metric spaces are proved. Other results are obtained in [9] and [10].

The notion of well posedness of fixed point problem have generate more interest to several mathematicians, for example in [12], [18], [38].

Several classical fixed point theorems and common fixed point theorems have been unified considering a general condition by an implicit relation in [29], [30] and in other papers. Recently, the method is used in the study of fixed points in metric spaces, symmetric spaces, quasi - metric spaces, $b$ - metric spaces, ultra - metric

Date: Received: November 6, 2014; Accepted: March 31, 2015.

2000 Mathematics Subject Classification. 54H25, 47H10.

Key words and phrases. Fixed point, $G_{p}$ - metric spaces, Well posedness of fixed point problem, Implicit relation. 
spaces, convex metric spaces, reflexive spaces, compact metric spaces, paracompact metric spaces, in two and three metric spaces, for single - valued mappings, hybrid pairs of mappings and set - valued mappings. Recently, the method is used in the study of fixed points for mappings satisfying contractive/extensive conditions of integral type, in fuzzy metric spaces, probabilistic metric spaces and intuitionistic metric spaces. Also, the method allows the study of local and global properties of fixed point structures.

The study of fixed points for mappings in $G$ - metric spaces is initiated in [33], [34], [35], [36], [37] and in other papers. The study of fixed point for mappings satisfying an implicit relation in partial metric spaces is initiated in [45].

In [3], [4], [5], [6], [31], [32] the authors studied well posedness of fixed point problem for mappings satisfying implicit relations.

The purpose of this paper is to prove a general fixed point theorem for mappings satisfying an implicit relation in $G_{p}$ - metric space. We prove that for these mappings, if $G_{p}$ - metric is symmetric, the fixed point problem is well posed.

\section{Preliminaries}

Definition 2.1 ([28], [46]). Let $X$ be a nonempty set. A function $G: X^{3} \rightarrow \mathbb{R}_{+}$is called a $G_{p}$ - metric on $X$ if the following conditions are satisfied:

$\left(G P_{1}\right): x=y=z$ if $G_{p}(x, y, z)=G_{p}(x, x, x)=G_{p}(y, y, y)=G_{p}(z, z, z)$,

$\left(G P_{2}\right): 0 \leq G_{p}(x, x, x) \leq G_{p}(x, x, y) \leq G_{p}(x, y, z)$ for all $x, y, z \in X$ with $y \neq z$,

$\left(G P_{3}\right): G_{p}(x, y, z)=G_{p}(y, z, x)=\ldots$ (symmetry in all three variables),

$\left(G P_{4}\right): G_{p}(x, y, z) \leq G_{p}(x, a, a)+G_{p}(a, y, z)-G_{p}(a, a, a)$ for all $x, y, z, a \in X$.

The pair $\left(X, G_{p}\right)$ is called a $G_{p}$ - metric space.

Definition $2.2([46])$. Let $\left(X, G_{p}\right)$ be a $G_{p}$ - metric space and $\left\{x_{n}\right\}$ a sequence in $X$. A point $x \in X$ is said to be the limit of the sequence $\left\{x_{n}\right\}$ or $x_{n} \rightarrow x\left(x_{n}\right.$ is $G_{p}$ - convergent to $x$ ) if $\lim _{m, n \rightarrow \infty} G_{p}\left(x, x_{n}, x_{m}\right)=G_{p}(x, x, x)$.

Theorem $2.1([8])$. Let $\left(X, G_{p}\right)$ be a $G_{p}$ - partial metric space. Then, for any $\left\{x_{n}\right\} \in X$ and $x \in X$, the following conditions are equivalent:

a) $\left\{x_{n}\right\}$ is $G_{p}$ - convergent to $x$,

b) $G_{p}\left(x_{n}, x_{n}, x\right) \rightarrow G_{p}(x, x, x)$ as $n \rightarrow \infty$,

c) $G_{p}\left(x_{n}, x, x\right) \rightarrow G_{p}(x, x, x)$ as $n \rightarrow \infty$.

Definition $2.3([46])$. Let $\left(X, G_{p}\right)$ be a $G_{p}$ - partial metric space.

1) A sequence $\left\{x_{n}\right\}$ of $X$ is called a $G_{p}$ - Cauchy sequence if $\lim _{m, n \rightarrow \infty} G_{p}\left(x_{n}, x_{m}, x_{m}\right)$ exists and is finite,

2) A $G_{p}$ - metric space is said to be $G_{p}$ - complete if and only if every $G_{p}$ Cauchy sequence in $X$ converges to $x \in X$ such that $\lim _{n, m \rightarrow \infty} G_{p}\left(x_{n}, x_{m}, x_{m}\right)=$ $G_{p}(x, x, x)$.

Lemma $2.1([8])$. Let $\left(X, G_{p}\right)$ be a $G_{p}$ - metric space. Then:

1) If $G_{p}(x, y, z)=0$ then $x=y=z$,

2) If $x \neq y$ then $G_{p}(y, x, x)>0$.

Definition 2.4 ([46]). A $G_{p}$ - metric on $X$ is said to be symmetric if $G_{p}(x, y, y)=$ $G_{p}(y, x, x)$. In this case $\left(X, G_{p}\right)$ is said to be symmetric.

Lemma $2.2([8])$. Let $\left(X, G_{p}\right)$ be a $G_{p}$ - metric space and $\left\{x_{n}\right\}$ a sequence in $X$. Assume that $\left\{x_{n}\right\}$ is $G_{p}$ - convergent to a point $x \in X$ with $G_{p}(x, x, x)=0$. Then $\lim _{n \rightarrow \infty} G_{p}\left(x_{n}, y, y\right)=G_{p}(x, y, y)$ for all $y \in X$. 
Moreover, $\lim _{n, m \rightarrow \infty} G_{p}\left(x_{n}, x_{m}, x\right)=0$.

\section{IMPLICIT RELATIONS}

Definition 3.1. Let $\mathfrak{F}_{W}$ be the set of all continuous functions $F\left(t_{1}, \ldots, t_{5}\right): \mathbb{R}_{+}^{5} \rightarrow \mathbb{R}$ satisfying

$\left(F_{1}\right): F$ is non - increasing in variable $t_{3}, t_{4}, t_{5}$,

$\left(F_{2}\right)$ : There exists $h_{1} \in[0,1)$ such that for all $u, v \geq 0, F(u, v, v, u, u+v) \leq 0$ implies $u \leq h_{1} v$,

$\left(F_{3}\right):$ There exists $h_{2} \in[0,1)$ such that for all $t, t^{\prime}>0, F\left(t, t, t^{\prime}, t, t+t^{\prime}\right) \leq 0$ implies $t \leq h_{2} t^{\prime}$.

In the following examples, property $\left(F_{1}\right)$ is obviously.

Example 3.1. $F\left(t_{1}, \ldots, t_{5}\right)=t_{1}-a t_{2}-b t_{3}-c t_{4}-d t_{5}$, where $a, b, c, d \geq 0$ and $a+b+c+2 d<1$.

$\left(F_{2}\right)$ : Let $u, v \geq 0$ be and $F(u, v, v, u, u+v)=u-a v-b v-c u-d(u+v) \leq 0$ which implies $u \leq h_{1} v$, where $0 \leq h_{1}=\frac{a+b+d}{1-(c+d)}<1$.

$\left(F_{3}\right)$ : Let $t, t^{\prime}>0$ be and $F\left(t, t, t^{\prime}, t, t+t^{\prime}\right)=t-a t-b t^{\prime}-c t-d\left(t+t^{\prime}\right) \leq 0$ which implies $t \leq h_{2} t^{\prime}$, where $0<h_{2}=\frac{b+d}{1-(a+c+d)}<1$.

Example 3.2. $F\left(t_{1}, \ldots, t_{5}\right)=t_{1}-k \max \left\{t_{2}, t_{3}, t_{4}, \frac{t_{5}}{2}\right\}$, where $k \in(0,1)$.

$\left(F_{2}\right)$ : Let $u, v \geq 0$ be and $F(u, v, v, u, u+v)=u-k \max \left\{u, v, \frac{u+v}{2}\right\} \leq 0$. If $u>v$, then $u(1-k) \leq 0$, a contradiction. Hence, $u \leq v$ which implies $u \leq h_{1} v$, where $0 \leq h_{1}=k<1$.

$\left(F_{3}\right)$ : Let $t, t^{\prime}>0$ be and $F\left(t, t, t^{\prime}, t, t+t^{\prime}\right)=t-k \max \left\{t, t^{\prime}, \frac{t+t^{\prime}}{2}\right\} \leq 0$. As in $\left(F_{2}\right)$ it follows that $t \leq h_{2} t^{\prime}$, where $0<h_{2}=k<1$.

Example 3.3. $F\left(t_{1}, \ldots, t_{5}\right)=t_{1}-k \max \left\{t_{2}, t_{3}, t_{4}, t_{5}\right\}$, where $k \in\left(0, \frac{1}{2}\right)$.

$\left(F_{2}\right)$ : Let $u, v \geq 0$ be and $F(u, v, v, u, u+v)=u-k(u+v) \leq 0$ which implies $u \leq h_{1} v$, where $0 \leq h_{1}=\frac{k}{1-k}<1$.

$\left(F_{3}\right)$ : Let $t, t^{\prime}>0$ be and $F\left(t, t, t^{\prime}, t, t+t^{\prime}\right)=t-k\left(t+t^{\prime}\right) \leq 0$ which implies $t \leq h_{2} t^{\prime}$, where $0<h_{2}=\frac{k}{1-k}<1$.

Example 3.4. $F\left(t_{1}, \ldots, t_{5}\right)=t_{1}-a t_{2}-b \max \left\{t_{3}, t_{4}\right\}-c t_{5}$, where $a, b, c \geq 0$ and $a+b+2 c<1$.

$\left(F_{2}\right)$ : Let $u, v \geq 0$ be and $F(u, v, v, u, u+v)=u-a v-b \max \{u, v\}-c(u+v) \leq 0$. If $u>v$, then $u[1-(a+b+2 c)] \leq 0$, a contradiction. Hence $u \leq v$, which implies $u \leq h_{1} v$, where $0 \leq h_{1}=\frac{a+b+c}{1-c}<1$.

$\left(F_{3}\right)$ : Let $t, t^{\prime}>0$ be and $F\left(t, t, t^{\prime}, t, t+t^{\prime}\right)=t-a t-b \max \left\{t, t^{\prime}\right\}-c\left(t+t^{\prime}\right) \leq 0$. Similar as in $\left(F_{2}\right)$, we obtain $0<h_{2}=\frac{b+c}{1-(a+c)}<1$.

Example 3.5. $F\left(t_{1}, \ldots, t_{5}\right)=t_{1}^{2}-a t_{2} t_{3}-b t_{4}^{2}-c t_{5}^{2}$, where $a, b, c \geq 0$ and $a+b+4 c<1$.

$\left(F_{2}\right)$ : Let $u, v \geq 0$ be and $F(u, v, v, u, u+v)=u^{2}-a v^{2}-b v^{2}-c(u+v)^{2} \leq 0$. If $u>v$, then $u^{2}[1-(a+b+4 c)] \leq 0$, a contradiction. Hence $u \leq v$, which implies $u \leq h_{1} v$, where $0 \leq h_{1}=\sqrt{a+b+4 c}<1$.

$\left(F_{3}\right)$ : Let $t, t^{\prime}>0$ be and $F\left(t, t, t^{\prime}, t, t+t^{\prime}\right)=t^{2}-a t t^{\prime}-b t^{2}-c\left(t+t^{\prime}\right)^{2} \leq 0$. If $t>t^{\prime}$ then $t^{2}[1-(a+b+4 c)] \leq 0$, a contradiction. Hence $t \leq t^{\prime}$, which implies $t \leq h_{2} t^{\prime}$, where $0<h_{2}=\sqrt{a+b+4 c}<1$.

Example 3.6. $F\left(t_{1}, \ldots, t_{5}\right)=t_{1}-a t_{2}-b t_{3}-c \max \left\{2 t_{4}, t_{5}\right\}$, where $a, b, c \geq 0$ and $a+b+2 c<1$. 
$\left(F_{2}\right)$ : Let $u, v \geq 0$ be and $F(u, v, v, u, u+v)=u-a v-b v-c \max \{2 u, u+v\} \leq 0$. If $u>v$, then $u[1-(a+b+2 c)] \leq 0$, a contradiction. Hence $u \leq v$, which implies $u \leq h_{1} v$, where $0 \leq h_{1}=a+b+2 c<1$.

$\left(F_{3}\right)$ : Let $t, t^{\prime}>0$ be and $F\left(t, t, t^{\prime}, t, t+t^{\prime}\right)=t-a t-b t^{\prime}-c \max \left\{2 t, t+t^{\prime}\right\} \leq 0$. If $t>t^{\prime}$ then $t[1-(a+b+2 c)] \leq 0$, a contradiction. Hence $t \leq t^{\prime}$, which implies $t \leq h_{2} t^{\prime}$, where $0<h_{2}=a+b+2 c<1$.

Example 3.7. $F\left(t_{1}, \ldots, t_{5}\right)=t_{1}^{2}-t_{1}\left(a t_{2}+b t_{3}+c t_{4}\right)-d t_{5}^{2}$, where $a>0, b, c, d \geq 0$ and $a+b+c+4 d<1$.

$\left(F_{2}\right)$ : Let $u, v \geq 0$ be and $F(u, v, v, u, u+v)=u^{2}-u(a v+b v+c u)-d(u+v)^{2} \leq 0$. If $u>v$, then $u^{2}[1-(a+b+c+4 d)] \leq 0$, a contradiction. Hence $u \leq v$, which implies $u \leq h_{1} v$, where $0 \leq h_{1}=\sqrt{a+b+c+4 d}<1$.

$\left(F_{3}\right)$ : Let $t, t^{\prime}>0$ be and $F\left(t, t, t^{\prime}, t, t+t^{\prime}\right)=t^{2}-t\left(a t+b t^{\prime}+c t\right)-d\left(t+t^{\prime}\right)^{2} \leq 0$. As in $\left(F_{2}\right)$ we obtain $t \leq h_{2} t^{\prime}$, where $0<h_{2}=\sqrt{a+b+c+4 d}<1$.

Example 3.8. $F\left(t_{1}, \ldots, t_{5}\right)=t_{1}-a t_{2}-b t_{3}-c \max \left\{2 t_{4}+t_{5}, t_{1}+t_{4}+t_{5}\right\}$, where $a \geq 0, b>0, c \geq 0$ and $a+b+4 c<1$.

$\left(F_{2}\right)$ : Let $u, v \geq 0$ be and $F(u, v, v, u, u+v)=u-a v-b v-c(3 u+v) \leq 0$ which implies $u \leq h_{1} v$, where $0 \leq h_{1}=\frac{a+b+c}{1-3 c}<1$.

$\left(F_{3}\right)$ : Let $t, t^{\prime}>0$ be and $F\left(t, t, t^{\prime}, t, t+t^{\prime}\right)=t-a t-b t^{\prime}-c\left(3 t+t^{\prime}\right) \leq 0$ which implies $t \leq h_{2} t^{\prime}$, where $0<h_{2}=\frac{b+c}{1-(a+3 c)}<1$.

\section{FIXED POINT THEOREMS}

Theorem 4.1. Let $\left(X, G_{p}\right)$ be a $G_{p}$ - metric space and let $T: X \rightarrow X$ be a mapping such that:

$$
\begin{gathered}
F\left(G_{p}(T x, T y, T y), G_{p}(x, y, y), G_{p}(x, T x, T x),\right. \\
\left.G_{p}(y, T y, T y), G_{p}(x, T y, T y)+G_{p}(y, T x, T x)\right) \leq 0
\end{gathered}
$$

for all $x, y \in X$, where $F$ satisfy property $\left(F_{3}\right)$. Then, $T$ has at most a fixed point.

Proof. Suppose that $T$ has two distinct fixed points $u$ and $v$. Then, by (4.1) we have successively

By $\left(G P_{2}\right)$,

$$
\begin{gathered}
F\left(G_{p}(T u, T v, T v), G_{p}(u, v, v), G_{p}(u, T u, T u),\right. \\
\left.G_{p}(v, T v, T v), G_{p}(u, T v, T v)+G_{p}(v, T u, T u)\right) \leq 0, \\
F\left(G_{p}(u, v, v), G_{p}(u, v, v), G_{p}(u, u, u),\right. \\
\left.G_{p}(v, v, v), G_{p}(u, v, v)+G_{p}(v, u, u)\right) \leq 0 .
\end{gathered}
$$

and

$$
G_{p}(u, u, u) \leq G_{p}(v, u, u)
$$

By $\left(F_{1}\right)$ we have

$$
G_{p}(v, v, v) \leq G_{p}(u, v, v)
$$

$$
\begin{gathered}
F\left(G_{p}(u, v, v), G_{p}(u, v, v), G_{p}(v, u, u),\right. \\
\left.G_{p}(u, v, v), G_{p}(u, v, v)+G_{p}(v, u, u)\right) \leq 0 .
\end{gathered}
$$

By $\left(F_{3}\right)$ we obtain

Similarly, we obtain

$$
G_{p}(u, v, v) \leq h_{2} G_{p}(v, u, u)
$$

$$
G_{p}(v, u, u) \leq h_{2} G_{p}(u, v, v) .
$$


Hence

a contradiction.

$$
G_{p}(u, v, v)\left(1-h_{2}^{2}\right) \leq 0,
$$

Hence, we get $u=v$ by using Lemma 2.1.

Theorem 4.2. Let $\left(X, G_{p}\right)$ be a $G_{p}$ - complete metric space and let $T: X \rightarrow X$ be a mapping satisfying inequality (4.1), for all $x, y \in X$, where $F \in \mathfrak{F}_{W}$. Then, $T$ has a unique fixed point.

Proof. Let $x_{0} \in X$ be an arbitrary point of $X$. We define $x_{n}=T x_{n-1}, n=1,2, \ldots$ . Then by (4.1) we have successively

$$
\begin{gathered}
F\left(G_{p}\left(T x_{n-1}, T x_{n}, T x_{n}\right), G_{p}\left(x_{n-1}, x_{n}, x_{n}\right), G_{p}\left(x_{n-1}, T x_{n-1}, T x_{n-1}\right),\right. \\
\left.G_{p}\left(x_{n}, T x_{n}, T x_{n}\right), G_{p}\left(x_{n-1}, T x_{n}, T x_{n}\right)+G_{p}\left(x_{n}, T x_{n-1}, T x_{n-1}\right)\right) \leq 0 \\
F\left(G_{p}\left(x_{n}, x_{n+1}, x_{n+1}\right), G_{p}\left(x_{n-1}, x_{n}, x_{n}\right), G_{p}\left(x_{n-1}, x_{n}, x_{n}\right),\right. \\
\left.G_{p}\left(x_{n}, x_{n+1}, x_{n+1}\right), G_{p}\left(x_{n-1}, x_{n+1}, x_{n+1}\right)+G_{p}\left(x_{n}, x_{n}, x_{n}\right)\right) \leq 0 .
\end{gathered}
$$

By $\left(G P_{4}\right)$ we have

$G_{p}\left(x_{n-1}, x_{n+1}, x_{n+1}\right) \leq G_{p}\left(x_{n-1}, x_{n}, x_{n}\right)+G_{p}\left(x_{n}, x_{n+1}, x_{n+1}\right)-G_{p}\left(x_{n}, x_{n}, x_{n}\right)$.

By $\left(F_{1}\right)$ we obtain

$$
\begin{gathered}
F\left(G_{p}\left(x_{n}, x_{n+1}, x_{n+1}\right), G_{p}\left(x_{n-1}, x_{n}, x_{n}\right), G_{p}\left(x_{n-1}, x_{n}, x_{n}\right),\right. \\
\left.G_{p}\left(x_{n}, x_{n+1}, x_{n+1}\right), G_{p}\left(x_{n-1}, x_{n}, x_{n}\right)+G_{p}\left(x_{n}, x_{n+1}, x_{n+1}\right)\right) \leq 0 .
\end{gathered}
$$

By $\left(F_{2}\right)$ we have

$$
G_{p}\left(x_{n}, x_{n+1}, x_{n+1}\right) \leq h_{1} G_{p}\left(x_{n-1}, x_{n}, x_{n}\right) .
$$

Therefore,

$$
G_{p}\left(x_{n}, x_{n+1}, x_{n+1}\right) \leq h_{1} G_{p}\left(x_{n-1}, x_{n}, x_{n}\right) \leq \ldots \leq h_{1}^{n} G_{p}\left(x_{0}, x_{1}, x_{1}\right) .
$$

By (4.2) and $\left(G P_{4}\right)$ we obtain for $m>n$ that

$$
\begin{aligned}
G_{p}\left(x_{n}, x_{m}, x_{m}\right) \leq & G_{p}\left(x_{n}, x_{n+1}, x_{n+1}\right)+G_{p}\left(x_{n+1}, x_{n+2}, x_{n+2}\right)+ \\
& +\ldots+G_{p}\left(x_{m-1}, x_{m}, x_{m}\right) \\
\leq & h_{1}^{n}\left(1+h_{1}+\ldots+h_{1}^{m-1}\right) G_{p}\left(x_{0}, x_{1}, x_{1}\right) \\
\leq & \frac{h_{1}^{n}}{1-h_{1}} G_{p}\left(x_{0}, x_{1}, x_{1}\right) .
\end{aligned}
$$

Consequently,

$$
\lim _{n, m \rightarrow \infty} G\left(x_{n}, x_{m}, x_{m}\right)=0
$$

and thus $\left\{x_{n}\right\}$ is a $G_{p}$ - Cauchy sequence. Since $\left(X, G_{p}\right)$ is $G_{p}$ - complete metric space, there exists $z \in X$ such that

$$
\lim _{n, m \rightarrow \infty} G\left(x_{n}, x_{m}, x_{m}\right)=\lim _{n \rightarrow \infty} G\left(z, x_{n}, x_{n}\right)=G_{p}(z, z, z)=0 .
$$

We prove that $z$ is a fixed point of $T$.

By (4.1) we have successively

$$
\begin{gathered}
F\left(G_{p}\left(T x_{n}, T z, T z\right), G_{p}\left(x_{n}, z, z\right), G_{p}\left(x_{n}, T x_{n}, T x_{n}\right),\right. \\
\left.G_{p}(z, T z, T z), G_{p}\left(x_{n}, T z, T z\right)+G_{p}\left(z, T x_{n}, T x_{n}\right)\right) \leq 0, \\
F\left(G_{p}\left(T x_{n}, T z, T z\right), G_{p}\left(x_{n}, z, z\right), G_{p}\left(x_{n}, x_{n+1}, x_{n+1}\right),\right. \\
\left.G_{p}(z, T z, T z), G_{p}\left(x_{n}, T z, T z\right)+G_{p}\left(z, x_{n+1}, x_{n+1}\right)\right) \leq 0 .
\end{gathered}
$$


By Lemma 2.2, (4.2) and (4.3), letting $n$ tend to infinity we obtain

$$
F\left(G_{p}(z, T z, T z), 0,0, G_{p}(z, T z, T z), G_{p}(z, T z, T z)\right) \leq 0 .
$$

By $\left(F_{2}\right)$ we obtain $G(z, T z, T z)=0$. By Lemma $2.1(a)$, we obtain $z=T z$. Hence $z$ is a fixed point of $T$. By Theorem $4.1, z$ is the unique fixed point of $T$.

Corollary 4.1. Let $\left(X, G_{p}\right)$ be a complete $G_{p}$ - metric space such that

$$
\begin{gathered}
G_{p}(T x, T y, T y) \leq k \max \left\{G_{p}(x, y, y), G_{p}(x, T x, T x),\right. \\
\left.G_{p}(y, T y, T y), \frac{G_{p}(x, T y, T y)+G_{p}(y, T x, T x)}{2}\right\},
\end{gathered}
$$

where $k \in(0,1)$. Then $T$ has an unique fixed point.

Proof. The proof it follows by Theorem 4.1 and Example 3.2.

Example 4.1. Let $X=[0, \infty), G_{p}: X^{3} \rightarrow \mathbb{R}$ defined by $G_{p}(x, y, z)=\max \{x, y, z\}$. Then $\left(X, G_{p}\right)$ is $G_{p}$ - complete metric space. Let $T: X \rightarrow X$ be defined by $T x=\frac{x}{x+2}$.

Without loss of generality, we assume that $x \geq y$. Then

$$
G_{p}(T x, T y, T y)=\frac{x}{x+2} \leq \frac{x}{2}=\frac{1}{2} G_{p}(x, y, y) \leq k G_{p}(x, y, y),
$$

where $k \in\left[\frac{1}{2}, 1\right)$ which implies

$$
\begin{gathered}
G_{p}(T x, T y, T y) \leq k \max \left\{G_{p}(x, y, y), G_{p}(x, T x, T x),\right. \\
\left.G_{p}(y, T y, T y), \frac{G_{p}(x, T y, T y)+G_{p}(y, T x, T x)}{2}\right\} .
\end{gathered}
$$

By Corollary 4.1, $T$ has an unique fixed point $x=0$. Moreover, $G_{p}(0,0,0)=0$.

5. Well posedness problem of FiXed point In $G_{p}$ - MEtric SPACES

Definition $5.1([38])$. Let $(X, d)$ be a metric space $(X, d)$ and let $f:(X, d) \rightarrow$ $(X, d)$ be a mapping. The fixed point problem of $f$ is said to be well posed if:

1) $f$ has an unique fixed point $x_{0}$,

2) for any sequence $\left\{x_{n}\right\} \in X$ with $\lim _{n \rightarrow \infty} d\left(f x_{n}, x_{n}\right)=0$ we have $\lim _{n \rightarrow \infty} d\left(x_{n}, x_{0}\right)=$ 0 .

Definition 5.2. A function $F: \mathbb{R}_{+}^{5} \rightarrow \mathbb{R}$ has property $\left(F_{p}\right)$ if for all $u, v, w \geq 0$ and $F(u, v, 0, w, u+v) \leq 0$, there exists $p \in(0,1)$ such that $u \leq p \max \{v, w\}$.

Example 5.1. $F\left(t_{1}, \ldots, t_{5}\right)=t_{1}-a t_{2}-b t_{3}-c t_{4}-d t_{5}$, where $a, b, c, d \geq 0, a+c+2 d>$ 0 and $a+b+c+4 d<1$.

$\left(F_{p}\right)$ : $\quad$ Let $u, v, w \geq 0$ be such that $F(u, v, 0, w, u+v)=u-a v-c w-d(u+$ $v) \leq 0$. If $u>\max \{v, w\}$, then $u[1-(a+c+2 d)] \leq 0$, a contradiction. Hence $u \leq \max \{v, w\}$, which implies $u \leq p \max \{v, w\}$, where $0<p=a+c+2 d<1$.

Example 5.2. $F\left(t_{1}, \ldots, t_{5}\right)=t_{1}-k \max \left\{t_{2}, t_{3}, t_{4}, \frac{t_{5}}{2}\right\}$, where $k \in(0,1)$.

$\left(F_{p}\right)$ : $\quad$ Let $u, v, w \geq 0$ be such that $F(u, v, 0, w, u+v)=u-k \max \left\{v, w, \frac{u+v}{2}\right\} \leq$ 0 . If $u>\max \{v, w\}$, then $u(1-k) \leq 0$, a contradiction. Hence $u \leq \max \{v, w\}$, which implies $u \leq p \max \{v, w\}$, where $0<p=k<1$.

Example 5.3. $F\left(t_{1}, \ldots, t_{5}\right)=t_{1}-k \max \left\{t_{2}, t_{3}, t_{4}, t_{5}\right\}$, where $k \in\left(0, \frac{1}{2}\right)$.

$\left(F_{p}\right): \quad$ Let $u, v, w \geq 0$ be such that $F(u, v, 0, w, u+v)=u-k \max \{v, w, u+$ $v\} \leq 0$. If $u>\max \{v, w\}$, then $u(1-2 k) \leq 0$, a contradiction. Hence $u \leq$ $\max \{v, w\}$, which implies $u \leq p \max \{v, w\}$, where $0<p=2 k<1$. 
Example 5.4. $F\left(t_{1}, \ldots, t_{5}\right)=t_{1}-a t_{2}-b \max \left\{t_{3}, t_{4}\right\}-c t_{5}$, where $a, b, c \geq 0$ and $0<a+b+2 c<1$.

$\left(F_{p}\right)$ : $\quad$ Let $u, v, w \geq 0$ be such that $F(u, v, 0, w, u+v)=u-a v-b w-c(u+$ $v) \leq 0$. If $u>\max \{v, w\}$, then $u[1-(a+b+2 c)] \leq 0$, a contradiction. Hence $u \leq \max \{v, w\}$, which implies $u \leq p \max \{v, w\}$, where $0<p=a+b+2 c<1$.

Example 5.5. $F\left(t_{1}, \ldots, t_{5}\right)=t_{1}^{2}-a t_{2} t_{3}-b t_{4}^{2}-c t_{5}^{2}$, where $a, b, c \geq 0, a+2 c>0$ and $a+b+2 c<1$

$\left(F_{p}\right)$ : $\quad$ Let $u, v, w \geq 0$ be such that $F(u, v, 0, w, u+v)=u^{2}-b w^{2}-c(u+v)^{2} \leq$ 0 . If $u>\max \{v, w\}$, then $u[1-(b+4 c)] \leq 0$, a contradiction. Hence $u \leq \max \{v, w\}$, which implies $u \leq p \max \{v, w\}$, where $0<p=b+4 c<1$.

Example 5.6. $F\left(t_{1}, \ldots, t_{5}\right)=t_{1}-a t_{2}-b t_{3}-c \max \left\{2 t_{4}, t_{5}\right\}$, where $a, b, c \geq 0$, $a+2 c>0$ and $a+b+2 c<1$.

$\left(F_{p}\right)$ : $\quad$ Let $u, v, w \geq 0$ be such that $F(u, v, 0, w, u+v)=u-a v-c \max \{2 w, u+$ $v\} \leq 0$. If $u>\max \{v, w\}$, then $u[1-(a+2 c)] \leq 0$, a contradiction. Hence $u \leq \max \{v, w\}$, which implies $u \leq p \max \{v, w\}$, where $0<p=a+2 c<1$.

Example 5.7. $F\left(t_{1}, \ldots, t_{5}\right)=t_{1}^{2}-t_{1}\left[a t_{2}+b t_{3}+c t_{4}\right]-d t_{5}^{2}$, where $a, b, c, d \geq 0$ and $0<a+c+4 d<1$.

$\left(F_{p}\right): \quad$ Let $u, v, w \geq 0$ be such that $F(u, v, 0, w, u+v)=u^{2}-u[a v+c w]-d(u+$ $v)^{2} \leq 0$. If $u>\max \{v, w\}$, then $u^{2}[1-(a+c+4 d)] \leq 0$, a contradiction. Hence $u \leq \max \{v, w\}$, which implies $u \leq p \max \{v, w\}$, where $0<p=a+c+4 d<1$.

Example 5.8. $F\left(t_{1}, \ldots, t_{5}\right)=t_{1}-a t_{2}-b t_{3}-c \max \left\{2 t_{4}+t_{5}, t_{1}+t_{4}+t_{5}\right\}$, where $a, b, c \geq 0, a+4 c>0$ and $a+b+4 c<1$.

$\left(F_{p}\right)$ : $\quad$ Let $u, v, w \geq 0$ be such that $F(u, v, 0, w, u+v)=u-a v-c \max \{2 w+$ $u+v, 2 u+w+v\} \leq 0$. If $u>\max \{v, w\}$, then $u[1-(a+4 c)] \leq 0$, a contradiction. Hence $u \leq \max \{v, w\}$, which implies $u \leq p \max \{v, w\}$, where $0<p=a+4 c<1$.

Definition 5.3. Let $\left(X, G_{p}\right)$ be a $G_{p}$ - metric space and let $T: X \rightarrow X$ be a function. The fixed point of $T$ is well posed if

1) $T$ has an unique fixed point $x_{0}$,

2) for any sequence $\left\{x_{n}\right\} \in X$ with $\lim _{n \rightarrow \infty} G_{p}\left(x_{n}, T x_{n}, T x_{n}\right)=0$ we have $\lim _{n \rightarrow \infty} G_{p}\left(x_{0}, x_{n}, x_{n}\right)=0$.

Theorem 5.1. Let $\left(X, G_{p}\right)$ be a $G_{p}$ - symmetric space and $T: X \rightarrow X$ a function satisfying the conditions of Theorem 4.2, where $F$ satisfy property $\left(F_{p}\right)$. Then the fixed point problem of $T$ is well posed.

Proof. By Theorem 4.2, T has an unique fixed point $x_{0}$. Let $\left\{x_{n}\right\}$ be a sequence in $X$ such that $\lim _{n \rightarrow \infty} G_{p}\left(x_{n}, T x_{n}, T x_{n}\right)=0$. By (4.1) we have successively

$$
\begin{gathered}
F\left(G_{p}\left(T x_{0}, T x_{n}, T x_{n}\right), G_{p}\left(x_{0}, x_{n}, x_{n}\right), G_{p}\left(x_{0}, T x_{0}, T x_{0}\right),\right. \\
\left.G_{p}\left(x_{n}, T x_{n}, T x_{n}\right), G_{p}\left(x_{0}, T x_{n}, T x_{n}\right)+G_{p}\left(x_{n}, T x_{0}, T x_{0}\right)\right) \leq 0, \\
F\left(G_{p}\left(x_{0}, T x_{n}, T x_{n}\right), G_{p}\left(x_{0}, x_{n}, x_{n}\right), 0,\right. \\
\left.G_{p}\left(x_{n}, T x_{n}, T x_{n}\right), G_{p}\left(x_{0}, T x_{n}, T x_{n}\right)+G_{p}\left(x_{n}, x_{0}, x_{0}\right)\right) \leq 0 .
\end{gathered}
$$

Since $\left(X, G_{p}\right)$ is symmetric, then $G\left(x_{n}, x_{0}, x_{0}\right)=G\left(x_{0}, x_{n}, x_{n}\right)$. Hence,

$$
\begin{gathered}
F\left(G_{p}\left(x_{0}, T x_{n}, T x_{n}\right), G_{p}\left(x_{0}, x_{n}, x_{n}\right), 0\right. \\
\left.G_{p}\left(x_{n}, T x_{n}, T x_{n}\right), G_{p}\left(x_{0}, T x_{n}, T x_{n}\right)+G_{p}\left(x_{0}, x_{n}, x_{n}\right)\right) \leq 0 .
\end{gathered}
$$


Since $F$ satisfy property $\left(F_{p}\right)$ then

$$
\begin{gathered}
G_{p}\left(x_{0}, T x_{n}, T x_{n}\right) \leq p \max \left\{G_{p}\left(x_{0}, x_{n}, x_{n}\right), G_{p}\left(x_{n}, T x_{n}, T x_{n}\right)\right\} \\
\leq p \max \left\{G_{p}\left(x_{0}, x_{n}, x_{n}\right)+G_{p}\left(x_{n}, T x_{n}, T x_{n}\right)\right\} .
\end{gathered}
$$

By $\left(G P_{4}\right)$ :

$$
\begin{aligned}
G_{p}\left(x_{0}, x_{n}, x_{n}\right) & \leq G_{p}\left(x_{0}, T x_{n}, T x_{n}\right)+G_{p}\left(T x_{n}, x_{n}, x_{n}\right) \\
& \leq p\left[G_{p}\left(x_{0}, x_{n}, x_{n}\right)+G_{p}\left(x_{n}, T x_{n}, T x_{n}\right)\right]+G_{p}\left(x_{n}, T x_{n}, T x_{n}\right),
\end{aligned}
$$

which implies

$$
G_{p}\left(x_{0}, x_{n}, x_{n}\right) \leq \frac{1+p}{1-p} G_{p}\left(x_{n}, T x_{n}, T x_{n}\right)
$$

Hence,

$$
\lim _{n \rightarrow \infty} G_{p}\left(x_{0}, x_{n}, x_{n}\right)=0
$$

and the fixed point problem of $T$ is well posed.

Corollary 5.1. Let $\left(X, G_{p}\right)$ be a $G_{p}$ - symmetric space and $T: X \rightarrow X$ be a function satisfying the conditions of Corollary 4.1. Then, the fixed point problem of $T$ is well posed.

\section{REFERENCES}

[1] Abbas, M., Nazi, T., Radanović, S., Some periodic point results in generalized metric spaces. Appl. Math. Comput. 217 (2010), 4084-4099.

[2] Abdeljawad, T., Karapinar, E., Tas, K., Existence and uniqueness of a common fixed point on partial metric spaces. Appl. Math. Lett. 24 (2011), no. 11, 1900-1904.

[3] Akkouchi, M., Popa, V., Well posedness of common fixed point problem for three mappings under strict contractive conditions. Bul. Univ. Petrol - Gaze Ploieşti, Ser. Mat. Inform. Fiz. 61 (2009), no. 2, 1-10.

[4] Akkouchi, M., Popa, V., Well posedness of a fixed point problem using $G$ - functions. Sci. Stud. Res. Ser. Math. Inform. 20 (2010), 5-12.

[5] Akkouchi, M., Popa, V., Well posedness of a fixed point problem for mappings satisfying an implicit relation. Demonstr. Math. 43 (2010), no. 4, 923-929.

[6] Akkouchi, M., Popa, V., Well posedness of fixed point problem for hybrid pairs of mappings. Fasc. Math. 46 (2011), 5-16.

[7] Altun, I., Sola, F., Simsek, H., Generalized contractions on partial metric spaces. Topology Appl. 157 (2010), no. 18, 2778-2785.

[8] Aydi, H., Karapinar, E., Salimi, P., Some fixed point results in $G_{p}$-metric spaces. J. Appl. Math. (2012), Article ID 891713.

[9] Barakat, M. A., Zidan, A. M., A common fixed point theorem for weak contractive maps in $G_{p}$ - metric spaces. J. Egyptian Math. Soc. (2014), DOI: 10.1016/j.joems.2014.06.008.

[10] Bilgili, N., Karapinar, E., Salimi, P., Fixed point theorems for generalized contractions on $G_{p}$ - metric spaces. J. Inequalities and Appl. Math.(2013), 2013:39.

[11] Chi, R., Karapinar, E., Thanh, T. D., A generalized contraction principle in partial metric spaces. Math. Comput. Modelling 55 (2012), no. 5 - 6, 1673-1681.

[12] De Blasi, F. S., Myjak, J., Sur la porosité de l'ensemble des contractions sans point fixe. Comptes Rend. Acad. Sci. Paris 308 (1989), 51-54.

[13] Dhage, B. C., Generalized metric spaces and mappings with fixed point. Bull. Calcutta Math. Soc. 84 (1992), 329-336.

[14] Dhage, B. C., Generalized metric spaces and topological structures I. An. Ştiinţ. Univ. Al. I. Cuza Iaşi, Mat. 46 (2000), 3-24.

[15] Kadelburg, Z., Nashine, H. K., Radanović, S., Fixed point results under various contractive conditions in partial metric spaces. RACSAM 10 (2013), 241-256.

[16] Karapinar, E., Erhan, I. M., Fixed point theorems for operators on partial metric spaces. Appl. Math. Lett. 24 (2011), no. 11, 1900-1904. 
[17] Kaushal, D. S., Pogey, S. S., Some results of fixed point theorems on complete $G$ - metric spaces. South Asian J. Math. 2 (2014), no. 4, 318-324.

[18] Lahiri, B. K., Das, P., Well-posedness and porosity of certain classes of operators. Demonstr. Math. 38 (2005), 170-176.

[19] Matthews, S., Partial metric topology and applications. Proc. 8th Summer Conference on General Topology and Applications, Ann. New York Acad. Sci. 728 (1994), 183-197.

[20] Mohanta, S., K., Some fixed point theorems in $G$ - metric spaces. An. Şt. Univ. Ovidius Constanţa, Ser. Mat. 20 (2012), no. 1, 285-306.

[21] Mustafa, Z., Khandagji, M., Shatanawi, W., Fixed point results on complete $G$ - metric spaces. St. Sci. Math. Hungarica 48 (2011), no. 3, 304-319.

[22] Mustafa, Z., Obiedat, H., A fixed point theorem of Reich in $G$ - metric spaces. Cubo Math. J. 12 (2010), no. 1, 83-93.

[23] Mustafa, Z., Obiedat, H., Awawdeh, F. Some fixed point theorem for mapping on complete $G$ - metric spaces. Fixed Point Theory Appl. (2008), Article ID 189870.

[24] Mustafa, Z., Shatanawi, W., Bataineh, M., Existence of fixed point results in $G$ - metric spaces. Intern. J. Math. Math. Sci. (2009), Article ID 283028.

[25] Mustafa, Z., Sims, B., Some remarks concerning D - metric spaces. Conf. Fixed Point Theory Appl., Yokohama (2004), 184-198.

[26] Mustafa, Z., Sims, B., A new approach to generalized metric spaces. J. Nonlinear Convex Anal. 7 (2006), no. 2, 289-297.

[27] Mustafa, Z., Sims, B., Fixed point theorems for contractive mappings in complete $G$ - metric spaces. Fixed Point Theory Appl. (2009), Article ID 917175.

[28] Parvaneh, V., Roshan, J. R., Kadelburg, Z., On generalized weakly GP-contractive mappings in ordered GP-metric spaces, Gulf J. Math. 1 (2013), 78-97.

[29] Popa, V., Fixed point theorems for implicit contractive mappings. Stud. Cercet. Ştiint., Ser. Mat., Univ. Bacău 7 (1997), 129-133.

[30] Popa, V., Some fixed point theorems for compatible mappings satisfying an implicit relation. Demonstr. Math. 32 (1999), 157-163.

[31] Popa, V., Well posedness of fixed point problem in orbitally complete metric spaces. Stud. Cercet. Ştiinţ., Ser. Mat., Univ. Bacău 16, Suppl. (2006), 209-214.

[32] Popa, V., Well posedness of fixed point problem in compact metric spaces. Bul. Univ. Petrol - Gaze Ploieşti, Ser. Mat. Inform. Fiz. 60 (2008), no. 1, 1-4.

[33] Popa, V., A general fixed point theorem for several mappings in $G$ - metric spaces. Sci. Stud. Res., Ser. Math. Inform. 21 (2011), no. 1, 205-214.

[34] Popa, V., Patriciu, A.-M., Two general fixed point theorems for pairs of weakly compatible mappings in G-metric spaces. Novi Sad J. Math. 42 (2012), no. 2, 49-60.

[35] Popa, V., Patriciu, A.-M., A general fixed point theorem for mappings satisfying an $\phi-$ implicit relation in complete $G$ - metric spaces. Gazi Univ. J. Sci. 25 (2012), no. 2, 403-408.

[36] Popa, V., Patriciu, A.-M., A general fixed point theorem for pair of weakly compatible mappings in $G$ - metric spaces. J. Nonlinear Sci. Appl. 5 (2012), 151-160.

[37] Popa, V., Patriciu, A.-M., Fixed point theorems for mappings satisfying an implicit relation in complete $G$ - metric spaces. Bul. Inst. Politehn. Iaşi, Ser. Mat. Mec. Teor. Fiz. 59 (63) (2013), 97-123.

[38] Reich, S., Zaslavski, A. J., Well posedness of fixed point problem. Far East J. Math. Special Volume, Part III (2001), 393-401.

[39] Shatanawi, W., Fixed point theory for contractive mappings satisfying $\varphi$ - maps in $G$ metric spaces. Fixed Point Theory Appl. (2010), Article ID 181650.

[40] Shatanawi, W., Common fixed point results for two self - maps in $G$ - metric spaces. Mat. Vesnik 65 (2013), no. 2, 143-150.

[41] Shatanawi, W., Chauhan, S., Postolache, M., Abbas, M., Radanović, S., Common fixed points for contractive mappings in $G$ - metric spaces. J. Adv. Math. Stud. 6 (2013), no. 1, 53-72.

[42] Shatanawi, W., Postolache, M., Some fixed point results for a $G$ - weak contraction in $G$ metric spaces. Abstr. Appl. Anal. (2012), Article ID 815870.

[43] Srivastava, R., Agrawal, S., Bhardwaj, R., Vardava, R., Fixed point theorems in complete $G$ - metric spaces. South Asian J. Math. 2 (2013), no. 2, 167-174.

[44] Vats, R. K., Kumar, S., Sihang, V., Fixed point theorems in complete $G$ - metric spaces. Fasc. Math. 47 (2011), 127-139. 
[45] Vetro, C., Vetro, F., Common fixed points of mappings satisfying implicit relations in partial metric spaces. J. Nonlinear Sci. Appl. 6 (2013), 152-161.

[46] Zand, M. R. A., Nezhad, A. N., A generalization of partial metric spaces. Appl. Math. 24 (2010), 86-93.

"VAsile Alecsandri" University of BacĂU, ROMANiA

E-mail address: vpopa@ub.ro

"Dunărea de Jos" University of Galaţi, Faculty of Sciences and Environment, Department of Mathematics and Computer Sciences, ROMANiA

E-mail address: Alina.Patriciu@ugal.ro 